Neurodegenerative Dis 2012;10:324-328

DOI: $\underline{10.1159 / 000332821}$
Received: June 30, 2011

Accepted after revision: September 2, 2011 Published online: December 7, 2011

\title{
Insights into Caspase-Mediated Apoptotic Pathways Induced by Amyloid- $\beta$ in Cerebral Microvascular Endothelial Cells
}

\author{
Silvia Fossati ${ }^{a}$ Jorge Ghiso ${ }^{a}$ b Agueda Rostagno $^{a}$ \\ Departments of a Pathology and ${ }^{b}$ Psychiatry, New York University School of Medicine, New York, N.Y., USA
}

\author{
Key Words \\ Cerebral amyloid angiopathy - Alzheimer's disease • \\ Endothelial cells $\cdot$ Apoptosis · Aggregation · Amyloid · \\ Caspases $\cdot$ Mitochondria
}

\begin{abstract}
Background: The vascular deposition of amyloid known as cerebral amyloid angiopathy (CAA) - an age-associated condition and a common finding in Alzheimer's disease - compromises cerebral blood flow, causing macro/microhemorrhages and/or cognitive impairment. Very little is known about the mechanisms causing CAA-related degeneration of cerebral vascular cells. The Dutch E22Q familial amyloid- $\beta$ $(A \beta)$ variant is primarily associated with $C A A$, and manifests clinically with severe cerebral hemorrhages. Objective: We aimed to determine the molecular mechanisms causing apoptosis of cerebral endothelial cells in the presence of wild-type A 340 or its vasculotropic E22Q variant. Methods: We challenged human brain microvascular endothelial cells with both $A \beta$ variants, and studied the apoptotic pathways triggered by these peptides. Results: Caspase-mediated apoptotic pathways were elicited by both peptides within time frames correlating with their aggregation properties and formation of oligomeric/protofibrillar assemblies. Our
\end{abstract}

\section{KARGER}

Fax +4161306 1234

E-Mail karger@karger.ch

www.karger.com
(C) 2011 S. Karger AG, Basel

Accessible online at: www.karger.com/ndd data revealed a primary activation of caspase-8 (typically triggered by death receptors) with secondary engagement of caspase-9, with cytochrome $C$ and apoptosis-inducing factor release from the mitochondria, suggesting the independent or synergistic engagement of extrinsic and intrinsic apoptotic mechanisms. Conclusion: Our data demonstrate the induction of caspase-8- and caspase-9-dependent mitochondrial-mediated apoptotic pathways by $A \beta$ oligomers/ protofibrils in vascular cells, likely implicating a primary activation of death receptors.

Copyright $\odot 2011$ S. Karger AG, Basel

\section{Introduction}

The deposition of amyloid in and around cerebral vessels is an often underestimated lesion that causes vascular damage, micro/macrohemorrhages and dysfunction of the entire neurovascular unit $[1,2]$. The main component of vascular deposits found in Alzheimer's disease (AD) brains is the 40 -amino-acid-long amyloid- $\beta$ (A $\beta 40)$ peptide. Mutations at positions $21-23$ or 34 generate $A \beta$ variants that associate with cerebral amyloid angiopathy (CAA), hemorrhagic stroke and dementia in early-onset familial forms of AD. The aggressive E22Q-A $\beta 40$ mutant 
causes cerebral hemorrhage in a disorder known as hereditary cerebral hemorrhage with amyloidosis Dutch type [3], characterized by recurrent strokes and vascular dementia in the absence of neurofibrillar pathology, with fatal cerebral bleeding resulting from the massive amyloid deposition in leptomeningeal and cortical vessels. Studies on the molecular pathogenesis of CAA and AD are currently centered on the structural changes affecting the respective amyloid subunits, with the transition to $\beta$ sheet-rich aggregated species being considered today as a key player in the disease process [4]. Insoluble amyloid species accumulate at the vascular walls, causing degeneration of the smooth muscle cell and endothelial cell (EC) layers while the presence of CAA and concomitant microhemorrhages in small capillary vessels lacking the smooth muscle cell layer emphasizes the relevance of studying the mechanism of $A \beta$-dependent cell death in brain ECs. Apoptosis has been reported as a major contributor to the cell death mechanisms associated with $\mathrm{AD}$, with increasing data from ours and other groups demonstrating the importance of intermediate $A \beta$ aggregates, in particular oligomers and protofibrils, for the mechanism of cytotoxicity $[5,6]$. However, the identification of the events triggering this apoptotic cascade is still a hotly debated issue. In the studies described herein, we establish a direct relationship between the aggregation state of the peptides deposited in sporadic and familial CAA cases, and their effect on EC death, with particular emphasis on the molecular events triggering the apoptotic machinery.

\section{Methods}

\section{Peptides}

Synthetic homologues of wild-type (WT) A 340 and the E22Q variant were synthesized using N-tert-butyloxycarbonyl chemistry by James I. Elliott at Yale University and purified by reversephase high-performance liquid chromatography on a Vydac C4 column (Western Analytical, Murrieta, Calif., USA) [7]. Peptides were dissolved to $1 \mathrm{mM}$ in hexafluoroisopropanol (Sigma). After a 1-hour incubation and lyophilization to remove hexafluoroisopropanol, peptides were thoroughly dissolved to $5 \mathrm{mM}$ in dimethyl sulfoxide, followed by the addition of deionized water and $2 \times$ PBS, $\mathrm{pH} 7.4$, to a final concentration of $1 \mathrm{mg} / \mathrm{ml}$ in PBS. Peptides were either incubated at $37^{\circ} \mathrm{C}$ for the aggregation studies or diluted into culture media at the required concentrations for the cell culture experiments.

\section{Electron Microscopy}

Peptides were placed onto carbon-coated 400-mesh $\mathrm{Cu} / \mathrm{Rh}$ grids (Ted Pella, Inc.) and stained with $1 \%$ uranyl acetate in distilled water (Polysciences, Inc.). Stained grids were examined in a
Philips CM-12 transmission electron microscope and photographed with a Gatan $(4 \mathrm{k} \times 4 \mathrm{k})$ digital camera at NYU School of Medicine Image Core Facility.

\section{Cell Cultures}

Immortalized human brain microvascular endothelial cells hCMEC/D3 [8] were cultured in complete EBM-2 medium (Lonza) containing growth supplements and $2.5 \%$ fetal bovine serum. Peptide treatments were performed in EBM-2 with $1 \%$ fetal bovine serum.

\section{Cell Death ELISA}

The extent of apoptosis caused by the different $A \beta$ peptides was assessed by quantitation of nucleosome formation with the Cell Death ELISA ${ }^{\text {plus }}$ kit (Roche Applied Science).

\section{Immunocytochemistry}

ECs were plated on glass chamber slides (Thermo Fisher Scientific), precoated with type I collagen. After 1 day in culture, cells were treated with the peptides, and subsequently fixed with $4 \%$ paraformaldehyde. Slides were separately incubated with monoclonal anti-cytochrome $\mathrm{C}$ (CytC) antibody (BD Biosciences), and polyclonal anti-apoptosis-inducing factor (AIF) antibody (Santa Cruz) followed, respectively, by Alexa Fluor 488-conjugated anti-mouse or anti-rabbit IgG (Invitrogen). Fluorescence was visualized with a Nikon Eclipse E 800 Deconvolution Microscope using Image-Pro Plus software (Media Cybernetics Inc., Bethesda, Md., USA) for image acquisition and processing and Autodeblur (Media Cybernetics) for 3-dimensional deconvolution.

\section{Caspase-3/7, -8, and -9 Activity Assays}

Caspase-3/7 activity was measured in cells treated with the $A \beta$ variants by Apo-ONE fluorescence assay (Promega) while caspase- 8 and -9 activation was evaluated by luminescent assays (Caspase-Glo 8 and Caspase-Glo 9, Promega). Fluorescence/luminescence were evaluated in a Synergy HT Multi-Mode Microplate Reader (Biotek).

\section{Results}

The aggregation status of $\mathrm{A} \beta-\mathrm{WT}$ and E22Q peptides was monitored by transmission electron microscopy (fig. 1a). After 1 day of incubation of $A \beta-W T$ at physiological salt concentrations, a few small globular species representing oligomeric conformations were observed, while E22Q presented abundant protofibrillar structures (up to $200 \mathrm{nM}$ in length) in combination with numerous oligomeric assemblies. After 3-day aggregation, the conformations present in the $\mathrm{A} \beta$-WT preparation were similar to the ones exhibited by E22Q at 1 day, whereas this variant displayed long fibrillar components together with the protofibrillar elements. To determine the proapoptotic effect of these peptides on ECs, we analyzed nucleosome formation by cell death ELISA (Roche). Apoptosis reached a 1.5-fold increase in cells treated for 


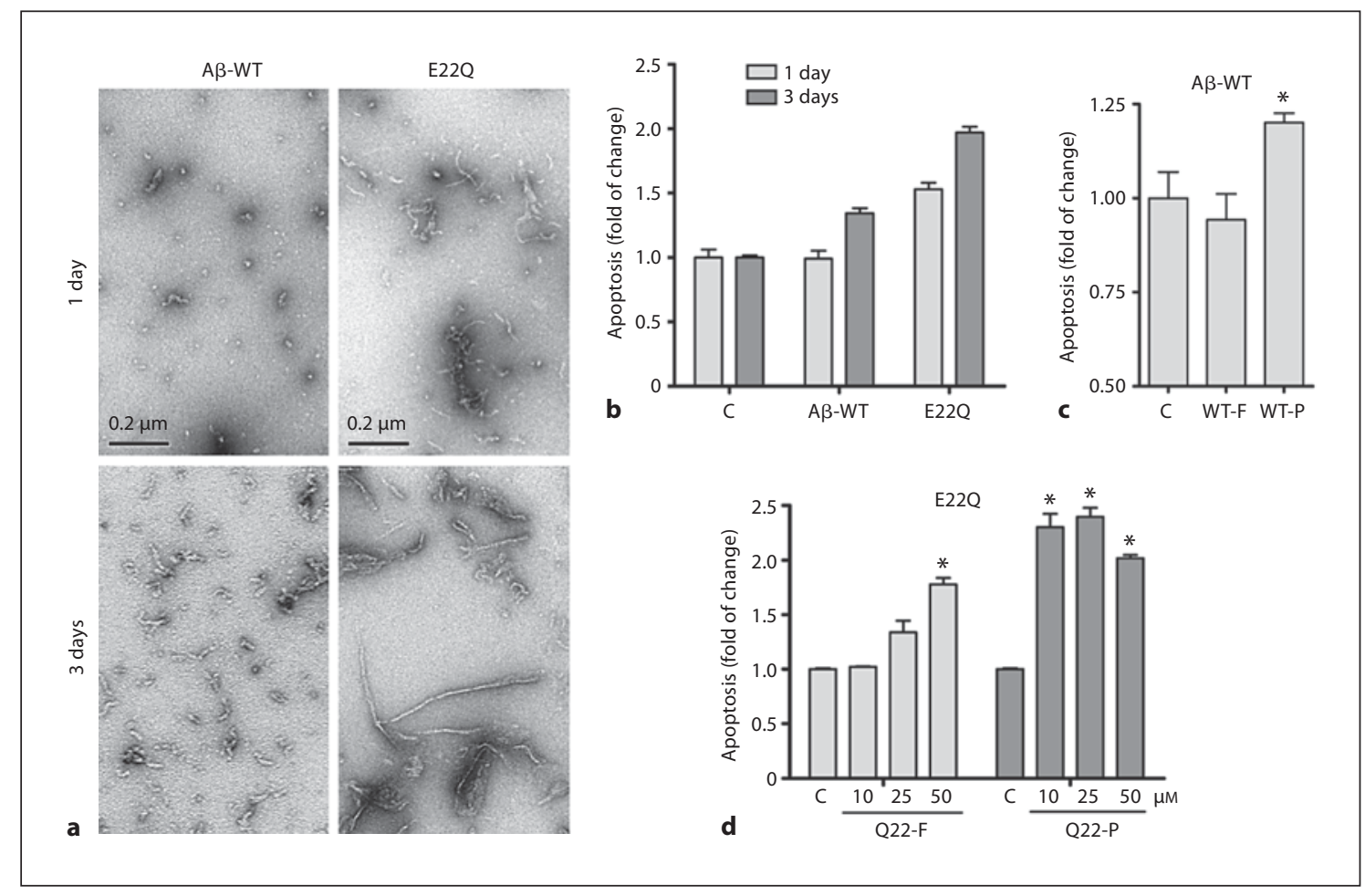

Fig. 1. Correlation between $A \beta$ peptide aggregation and toxicity for endothelial cells. a Electron microscopy studies showing the structural species present after 1 day and 3 days of incubation under physiological conditions in $\mathrm{A} \beta 40-\mathrm{WT}$ and $\mathrm{E} 22 \mathrm{Q}$ preparations. $\mathbf{b}$ Apoptosis induced by $A \beta$ variants. ECs were treated with $50 \mu \mathrm{M} \mathrm{A} \beta-\mathrm{WT}$ or E22Q for 1 or 3 days. c Apoptosis levels in ECs treated for $24 \mathrm{~h}$ with $50 \mu \mathrm{M} A \beta-\mathrm{WT}$, either freshly solubilized
(WT-F) or preaggregated for 2 days (WT-P). ${ }^{*} \mathrm{p} \leq 0.05$. d Apoptosis in ECs treated for $20 \mathrm{~h}$ with 10, 25, or $50 \mu \mathrm{M} \mathrm{E} 22 \mathrm{Q}$, either freshly solubilized (Q22-F) or preaggregated for 2 days (Q22-P). ${ }^{*} \mathrm{p} \leq 0.01$. Apoptosis in $\mathbf{b}-\mathbf{d}$ - evaluated as nucleosome formation assessed by cell death ELISA - is represented as fold of change compared to untreated controls.
1 day with E22Q peptide, while at the same time point $A \beta$-WT had no effect (fig. 1b). Levels of nucleosome formation after a 3-day treatment with $\mathrm{A} \beta$-WT were similar to those elicited by $A \beta-E 22 \mathrm{Q}$ after 1 day - correlating to the comparable aggregation species present in the two preparations at the respective time points - whereas ECs treated with E22Q peptide for 3 days presented a 2-fold increase in apoptosis compared to untreated controls (fig. 1b). To further establish whether intermediate aggregation species were responsible for the peptide toxicity, we measured nucleosome formation in cells treated for 1 day with $A \beta-W T$, either freshly solubilized or preaggregated for 2 days to allow the formation of oligomers/protofibrils. The level of apoptosis, while being comparable to the untreated controls in cells challenged with fresh peptide, was significantly elevated in cells treated with preaggregated peptide (fig. 1c). For the aggressive $A \beta-E 22 Q$, we evaluated apoptosis induction af- ter a 20 -hour challenge with 10,25 or $50 \mu \mathrm{M}$ fresh and preaggregated peptide. The 2-day preaggregation rendered the peptide more toxic for ECs, being the amount of nucleosomes in cells treated with each concentration of preaggregated E22Q higher in all cases than after treatment with fresh peptide (fig. 1d). To better understand the mechanism of $A \beta$-induced apoptosis in ECs, we performed immunocytochemistry experiments to analyze the possible release of the mitochondrial proteins CytC and AIF. Figure 2a shows that in control conditions CytC (top panels) and AIF (bottom panels) presented the punctuate distribution characteristic of mitochondrial localization. CytC and AIF were released into the cytoplasm after a 1- or 2-day challenge with E22Q, respectively, or after a 3-day treatment with both peptides, indicating that the two $A \beta$ variants induced similar apoptotic pathways, albeit within different time frames. The activation of different members of the cas- 

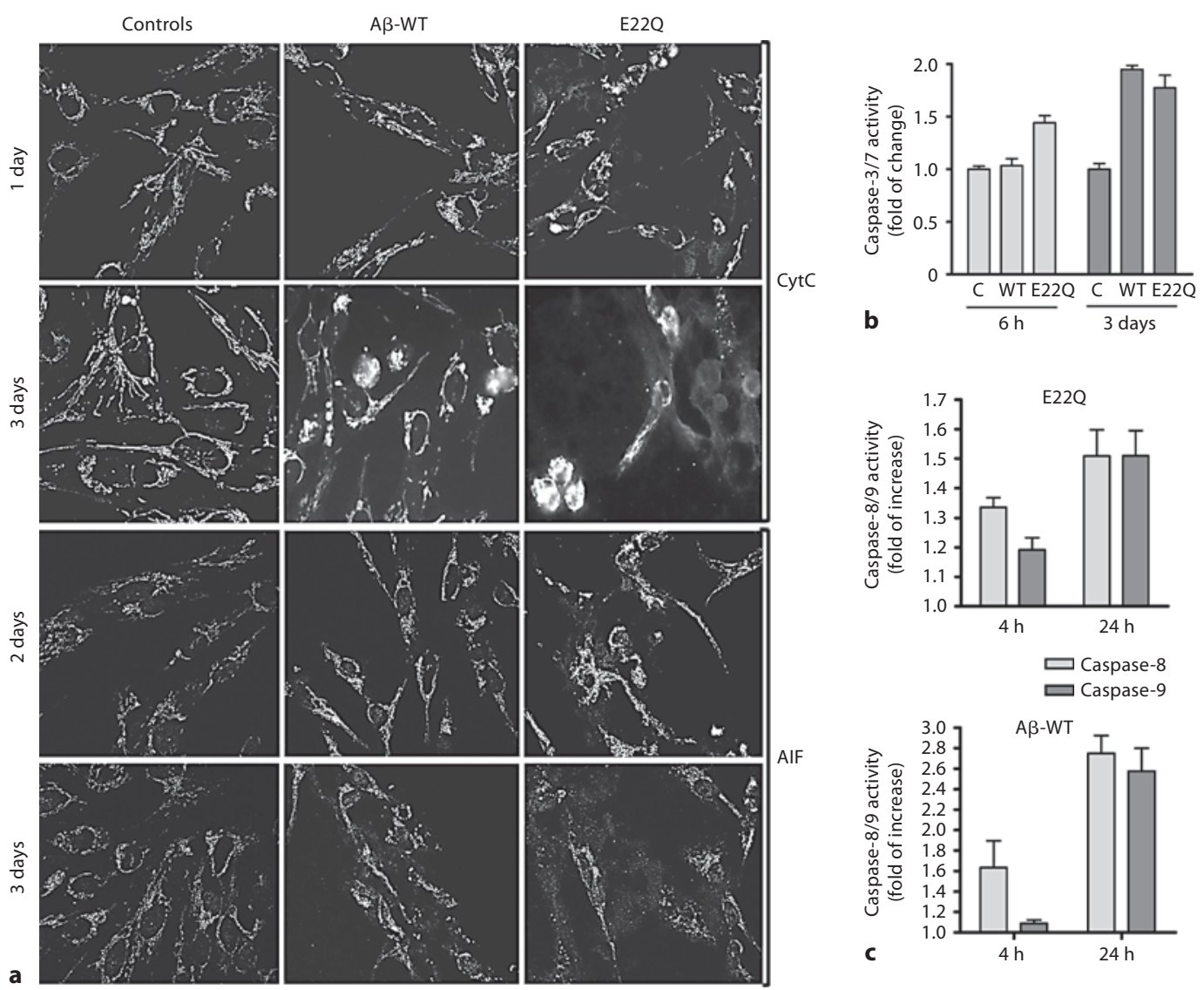

Fig. 2. Release of mitochondrial proteins and caspase activation in $\mathrm{A} \beta$-treated endothelial cells. a Immunocytochemistry visualizing CytC and AIF in ECs treated with $50 \mu \mathrm{M} \mathrm{A} \beta-\mathrm{WT}$ and E22Q (1- and 3-day treatment for CytC; 2- and 3-day treatment for AIF). Punctuate pattern denotes mitochondrial localization under control conditions, while decrease in mitochondrial staining and cytoplasmic localization is evident after 1-day E22Q challenge and 3 -day A $\beta$-WT treatment. b Caspase-3/7 activation was measured in EC after a 6-hour and 3-day treatment with A $\beta$-WT and E22Q. c Top panel: caspase- 8 (light gray bars) and caspase- 9 (dark gray bars) activity was measured in ECs treated for 4 and $24 \mathrm{~h}$ with freshly solubilized A $\beta$-E22Q. Bottom panel: caspase-8 (light gray bars) and caspase- 9 (dark gray bars) activity was measured in ECs treated for either 4 or $24 \mathrm{~h}$ with A $\beta$-WT preaggregated for 3 days. Caspase activity is represented as fold of change compared to untreated controls at the same time point. pase family was also investigated. Figure $2 \mathrm{~b}$ illustrates the activation of the effector caspase-3/7 in ECs challenged for $6 \mathrm{~h}$ or 3 days with the $A \beta$ peptides, compared to untreated controls at the same time points. E22Q induced caspase-3/7 activation after only a 6-hour treatment, while after 3 days both $\mathrm{A} \beta-\mathrm{WT}$ and E22Q induced an almost 2 -fold increase in caspase-3/7 activity. Activation of the initiator caspases- 8 and -9 , indicating the participation of extrinsic or intrinsic apoptotic pathways, respectively, was evaluated in ECs treated with fresh $A \beta E 22 Q$ (fig. $2 c$, top panel), or A $\beta$-WT preaggregated for 3 days (fig. 2c, bottom panel). In both cases, after a 4-hour treatment, caspase- 8 activity was higher than that of caspase-9, whereas after a 24 -hour peptide incubation the activity of both caspases was upregulated to similar levels. These results suggest the participation of extrinsic, death-receptor-mediated apoptotic pathways, initially involving activation of caspase-8, which, after cleavage of Bid and translocation of its truncated form to the mitochondria, induce the release of $\mathrm{CytC}$ and subsequent activation of caspase-9. 


\section{Conclusions}

The $A \beta 40-E 22 Q$ genetic variant exhibits enhanced aggregation kinetics, forming protofibrillar and fibrillar assemblies more rapidly than the WT A $\beta 40$ counterpart. The early stages of apoptosis in cerebral microvascular ECs precede fibril formation, correlating with the presence of intermediate-sized oligomeric and protofibrillar assemblies. $A \beta 40$ variants, albeit requiring different time frames for maximal effect, are strong inducers of the apoptotic mitochondrial pathway, triggering $\mathrm{CytC}$ and
AIF release from the mitochondria and activation of the effector caspases-3/7. The participation of both caspase- 8 and -9 , with a primary activation of caspase- 8 , indicates involvement of extrinsic death-receptor-mediated pathways in $A \beta$-induced brain vascular cell apoptosis.

\section{Acknowledgments}

This work was supported by NIH grants AG30539, NS051715, AG008051 ADC Pilot, the Alzheimer's Association, and the American Heart Association.

\section{References}

$>1$ Revesz T, Holton JL, Lashley T, Plant G, Frangione B, Rostagno A, Ghiso J: Genetics and molecular pathogenesis of sporadic and hereditary cerebral amyloid angiopathies. Acta Neuropathol 2009;118:115-130.

$>2$ Zlokovic BV: The blood brain barrier in health and chronic neurodegenerative disorders. Neuron 2008;57:178-201.

-3 Levy E, Carman MD, Fernandez Madrid IJ, Power MD, Lieberburg I, van Duinen SG, Frangione B: Mutation of the Alzheimer's disease amyloid gene in hereditary cerebral hemorrhage, Dutch type. Science 1990;248: 1124-1126.
4 Walsh DM, Selkoe DJ: A beta oligomers - A decade of discovery. J Neurochem 2007;101: 1172-1184.

5 Ono K, Condron MM, Teplow DB: Structure-neurotoxicity relationships of amyloid betaprotein oligomers. Proc Natl Acad Sci USA 2009; 106:14745-14750.

6 Fossati S, Cam J, Meyerson J, Mezhericher E, Romero IA, Couraud PO, Weksler BB, Ghiso J, Rostagno A: Differential activation of mitochondrial apoptotic pathways by vasculotropic amyloid-beta variants in cells composing the cerebral vessel walls. FASEB J 2010;24:229-241.
Miravalle L, Tokuda T, Chiarle R, Giaccone G, Bugiani O, Tagliavini F, Frangione B, Ghiso J: Substitutions at codon 22 of Alzheimer's $\mathrm{A} \beta$ peptide induce diverse conformational changes and apoptotic effects in human cerebral endothelial cells. J Biol Chem 2000; 275:27110-27116.

8 Weksler BB, Subileau EA, Perrière N, Charneau $\mathrm{P}$, Holloway $\mathrm{K}$, Leveque $\mathrm{M}$, TricoireLeignel H, Nicotra A, Bourdoulous S, Turowski P, Male DK, Roux F, Greenwood J, Romero IA, Couraud PO: Blood-brain barrier-specific properties of a human adult brain endothelial cell line. FASEB J 2005;19:18721874 